\title{
Cyclic performance of viscoelastic dielectric elastomers with solid hydrogel electrodes
}

\section{Citation}

Bai, Yuanyuan, Yanhui Jiang, Baohong Chen, Choon Chiang Foo, Yongcun Zhou, Feng Xiang, Jinxiong Zhou, Hong Wang, and Zhigang Suo. 2014. "Cyclic Performance of Viscoelastic Dielectric Elastomers with Solid Hydrogel Electrodes." Appl. Phys. Lett. 104 (6) (February 10): 062902. doi:10.1063/1.4865200.

\section{Published Version}

doi:10.1063/1.4865200

\section{Permanent link}

http://nrs.harvard.edu/urn-3:HUL.InstRepos:13910568

\section{Terms of Use}

This article was downloaded from Harvard University's DASH repository, and is made available under the terms and conditions applicable to Open Access Policy Articles, as set forth at http:// nrs.harvard.edu/urn-3:HUL.InstRepos:dash.current.terms-of-use\#OAP

\section{Share Your Story}

The Harvard community has made this article openly available.

Please share how this access benefits you. Submit a story.

\section{Accessibility}




\title{
Cyclic performance of viscoelastic dieletric elastomers with solid hydrogel electrodes
}

\author{
Yuanyuan Bai ${ }^{1}$, Yanhui Jiang ${ }^{2}$, Baohong Chen ${ }^{2}$, Choon Chiang Foo ${ }^{3}$, Feng Xiang ${ }^{1}$, \\ Jinxiong Zhou ${ }^{2}$, Hong Wang, a), and Zhigang Suo ${ }^{4, \text { a) }}$
}

${ }^{1}$ School of Electronics and Information Engineering, Electronic Materials Research Laboratory, Xi'an Jiaotong University, Xi'an 710049, China

${ }^{2}$ State Key Laboratory for Strength and Vibration of Mechanical Structures, International Center for Applied Mechanics and School of Aerospace, Xi'an Jiaotong University, Xi'an 710049, China

3 Institute of High Performance Computing, 1 Fusionopolis Way, \#16-16 Connexis, Singapore 138632, Singapore

4School of Engineering and Applied Sciences, Kavli Institute of Bionano Science and Technology, Harvard University, Cambridge, Massachusetts 02138, USA

Hydrogels containing electrolyte can work as ionic conductors to actuate dielectric elastomer (DE) artificial muscles. Based on a popular acrylic elastomer and an elegant design of a circular actuator, we study both theoretically and experimentally the cyclic performance of DE with solid hydrogel electrodes. The elasticity of solid electrodes constrains the maximum strain that is attainable for one cycle of triangular voltage, and it also diminishes the accumulated relaxation after many cycles of loadings.

Corresponding authors:

a) hwang@mail.xjtu.edu.cn, suo@seas.harvard.edu 
Dielectric elastmer (DE) has emerged as one of the competitive candidates for the realization of soft robotics, ${ }^{1-9}$ adaptive optics, ${ }^{10-12}$ actuators and sensors,,${ }^{9,13-22}$ and generators, ${ }^{23-26}$ due to its unique features such as low cost, ease of production line manufacture or even $3 \mathrm{D}$ printing, silentness and large deformation capability.4,9,13-16 Among various commercial dielectric elastomers, the VHB series acrylic elastomers by 3M company, are extensively used for prototyping or real-purpose device fabrication. This type of elastomer, however, has long been identified to be viscoelastic. Viscoelasticity of DE would affect the performance of DE devices and should be taken into account during the design stage. This aspect is crucial for applications involving dynamic input voltages, such as loudspeaker or vibrator. Cyclic behavior is also closely related to the lifetime of DE. Several independent theoretical and experimental studies regarding the viscoelastic or dynamic responses of $\mathrm{DE}$ parallel in the literature, ${ }^{27-35}$ whereas rigorous correlation between theory and experiment is lacking.

Conventional DE devices mainly use carbon grease compliant electrodes to drive the elastomer. Other fluidic or solid electrodes have also been developed. $9,33,36-40$ All these electrodes are electronic conductors. Very recent work reported that hydrogels containing electrolyte can work as ionic electrodes to actuate a DE loudspeaker capable of operation at frequencies beyond 10 kilohertz and voltages above 10 kilovolts. ${ }^{41}$ Motivated by this new discovery, this letter presents both experiment and analysis of cyclic viscoelastic behavior of DE actuators with solid hydrogel electrodes.

We adopt the circular configuration to investigate the performance of commercial VHB 4910 (thickness $1 \mathrm{~mm}$ ) from $3 \mathrm{M}$ company. Figure $1(\mathrm{a})$ and (b) shows the schematics of the top 
and side views of the experimental setup. A pair of solid hydrogel electrodes was glued on the center of the prestretched circular actuator and connected to external circuits. Cycles of triangular waveform voltages as shown in Figure 1(c) were supplied. The hydrogel electrode was synthesized following a standard procedure reported elsewhere ${ }^{42}$ by dissolving Acrylamide (AAm) monomer and lithium chloride ( $\mathrm{LiCl}$ ) powder into deionized water, and crosslinking AAm by N,N'-methylenebisacrylamide (MBAA). The obtained PAAm hydrogel containing LiCl is transparent, stretchable and conductive. The Young's modulus of the hydrogel is $1.8 \mathrm{kPa}$, the maximum rupture stretch is over 20 , and the measured conductivity is about

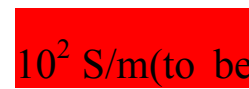

measured). Figure 1(d), (e) and (f) present the snapshots of DE with hydrogel electrodes corresponding to voltage-off state, voltage-on states for cycle 1 and cycle 30, respectively. As a comparison, Figure $1(\mathrm{~g})$, (h) and (i) present the snapshots of DE with carbon grease electrodes corresponding to voltage-off state, voltage-on states for cycle 1 and cycle 30, respectively.

The applied voltage given in Figure 1(c) ramps up from zero to its peak and then goes down to zero again to complete one cycle. During this process, the areal strain of the actuator increases firstly to reach its maximum strain and then decreases gradually. At the instant of voltage becomes zero, the elastomer retains a residual strain due to the viscoelasticity. The measured areal strain is recorded up to 30 cycles and three typical cycles are shown in Figure 2 (a). Given a fixed ramping velocity of applied voltage, say $V_{\text {ramp }}=250 \mathrm{OV} / \mathrm{s}$ in this case, the process in Figure 2(a) can be converted equivalently into a time history shown in Figure 2(b), whereby open blue circles denote experiment and red solid line is simulation. Figure 2(a) and (b) indicate that both residual and maximum strains increase as number of cycles increases, and eventually 
they reach their steady-state values.

The simulation result in Figure 2(b) is obtained by adopting the previously developed rheological models of $\mathrm{DE}^{43}$ but with incorporation of elasticity of solid electrode. 33 DE is approximated by a rheological model with two parallel units: one unit consists of a spring with shear modulus $\mu^{\alpha}$, and another unit consists of a spring with shear modulus $\mu^{\beta}$ and a dashpot with viscosity $\eta$. The solid hydrogel electrode is treated as a hyperelastic spring with shear modulus $\mu^{\gamma}$. The Gent model is used to represent all springs, with $J_{\lim }^{\alpha}, J_{\lim }^{\beta}, J_{\lim }^{\gamma}$ being three corresponding extension limits, respectively. 44 The original thickness of DE is $H_{o}$, and it becomes $H_{1}=H_{0} / \lambda_{p}^{2}$ after an equibiaxial prestretch, $\lambda_{p}$. The overall thickness of hydrogel electrodes are $H_{2}$, then the volume fraction of DE and electrodes are defined as $\phi_{1}=H_{1} /\left(H_{1}+H_{2}\right)$ and $\phi_{2}=1-\phi_{1}$, respectively. Incorporating the effects of electrode elasticity and assuming a homogeneous deformation state, the constitutive relation of the DE-hydrogel composite system reads

$\lambda_{p}^{-1} \sigma_{p} \lambda+\phi_{1} \varepsilon H_{0}^{-2} V^{2} \lambda^{4}=\phi_{1}\left[\frac{\mu^{\alpha}\left(\lambda^{2}-\lambda^{-4}\right)}{1-\left(2 \lambda^{2}+\lambda^{-4}-3\right) / J_{\lim }^{\alpha}}+\frac{\mu^{\beta}\left(\lambda^{2} \xi^{-2}-\lambda^{-4} \xi^{4}\right)}{1-\left(2 \lambda^{2} \xi^{-2}+\lambda^{-4} \xi^{4}-3\right) / J_{\lim }^{\beta}}\right]+\phi_{2} \frac{\mu^{\gamma}\left(\lambda^{2} \lambda_{p}^{-2}-\lambda^{-4} \lambda_{p}^{4}\right)}{1-\left(2 \lambda^{2} \lambda_{p}^{-2}+\lambda^{-4} \lambda_{p}^{4}-3\right) / J_{\lim }^{\gamma}},(1)$

where $\varepsilon=4.11 \times 10^{-11} \mathrm{~F} / \mathrm{m}$, the permittivity of $\mathrm{DE} ; \lambda$, the overall stretch of $\mathrm{DE}$ (the same as the stretch of spring $\alpha$ ); $\mathrm{V}$, applied voltage; $\sigma_{p}=\frac{\mu^{\alpha}\left(\lambda_{p}^{2}-\lambda_{p}^{-4}\right)}{1-\left(2 \lambda_{p}^{2}+\lambda_{p}^{-4}-3\right) / J_{\lim }^{\alpha}}$, the stress needed to attain a prestrech, $\lambda_{p}$, and set as a constant in simulation; $\xi$, the stretch in the dashpot. The stretch of spring $\beta$ is determined by adopting a multiplication rule as $\lambda / \xi$. The dashpot is modeled as a Newtonian fluid, and the rate of deformation is related to stress via 


$$
\frac{d \xi}{\xi d t}=\frac{1}{\eta} \frac{\mu^{\beta}\left(\lambda^{2} \xi^{-2}-\lambda^{-4} \xi^{4}\right)}{1-\left(2 \lambda^{2} \xi^{-2}+\lambda^{-4} \xi^{4}-3\right) / J_{\lim }^{\beta}} .
$$

Equation (1) recovers the relation for a DE with compliant electrode, e.g., carbon grease, by letting $\phi_{2}=0$ and $\phi_{1}=1$, where electrode thickness and the constraining effects of electrodes are negligible. The parameters used in our simulation is as follows: $\mu^{\alpha}=24.5 \mathrm{kPa}$, $\mu^{\beta}=40 \mathrm{kPa}, \mu^{\gamma}=0.6 \mathrm{kPa}, J_{\lim }^{\alpha}=110, J_{\lim }^{\beta}=55, J_{\lim }^{\gamma}=500, \eta=\tau_{V} \cdot \mu^{\beta}, \tau_{V}$ is a parameter reflecting the relaxation time, $\tau_{V}=400 \mathrm{~s}$.

It is intriguing to compare the cyclic performance of DE with hydrogel electrodes to that of DE with carbon grease electrodes. This comparison gives clearly the effects of elastomeric electrodes on the viscoelastic deformation of DE. Figure 3(a) plots both simulation and experimental results of DE with hydrogel and carbon grease electrodes. The ramping velocity is $25 \mathrm{OV} / \mathrm{s}$ and the amplitudes of applied voltage are $4 \mathrm{kV}$ for $0.5 \mathrm{~mm}$ thick hydrogel electrodes, and $3.5 \mathrm{kV}$ and $4 \mathrm{kV}$ for carbon grease electrodes. Even for a soft hydrogel electrode (Young's modulus $1.8 \mathrm{kPa}$ herein), its constraining effect is obvious: the plateau of the steady-state maximum strain of DE with hydrogel electrode at $4 \mathrm{kV}$ is less than half of that with carbon grease electrode at the same voltage; it is even less than that with carbon grease at $3.5 \mathrm{kV}$. Both experimental and simulation results prove this constraining effect. For not very large deformation, such as DE with hydrogel electrodes or DE with carbon grease electrodes working at not high voltage, e.g., less than $3.5 \mathrm{kV}$, there is a good agreement between the experiment and model prediction. For $\mathrm{DE}$ with carbon grease electrode at $4 \mathrm{kV}$, the deformation is pretty large. The constant approximation of mechanical loading, $\sigma_{p}$, does not hold any more. That is the 
reason why there is a discrepancy between theory and experiment. Rigorous calculation with a varying mechanical loading needs solution of a boundary-value problem, and the details can refer to reference. 33

Ramping velocity also affects the cyclic performance of DE, and systematic experiments for both hydrogel electrodes and carbon grease electrodes have been carried out. Figure 3(b) and (c) show the evolution of maximum strain for carbon grease electrodes and hydrogel electrodes, respectively. The peak value of the applied voltage is set at the same, $4 \mathrm{kV}$, but the ramping velocities are varied as $250 \mathrm{~V} / \mathrm{s}, 500 \mathrm{~V} / \mathrm{s}$ and $1000 \mathrm{~V} / \mathrm{s}$. The cyclic maximum strain evolves gradually, starting from the first maximum strain that is achieved after cycle 1 , to its steady-state value indicated by the plateau. The shift from the first maximum strain to the plateau of maximum strains depends on ramping velocity and property of electrodes. For carbon grease electrodes, the shifts from the first to the steady-state maximum strains are $0.26,0.17$ and 0.15 , respectively, corresponding to ramping velocities $250 \mathrm{~V} / \mathrm{s}, 500 \mathrm{~V} / \mathrm{s}$ and $1000 \mathrm{~V} / \mathrm{s}$. The decrease of shift to steady-state plateau with increasing ramping velocity is due to the relaxation of elastomer: If ramping velocity is low, the elastomer has enough time to relax when one cycle is completed, and the accumulated relaxation will become larger after many cycles; when applied voltage ramps up and down very quickly, the elastomer does not have enough time to response such that the relaxation does not fully develop and exhibits smaller shift. For hydrogel electrodes, however, the shifts are about 0.06 0.07, approximately constant for the three ramping velocities. This implies that the elasticity of solid electrodes diminishes the viscoelastic deformation of DE. 
In summary, we study both theoretically and experimentally the cyclic performance of an acrylic elastomer. Polyacrylamide hydrogels containing $\mathrm{LiCl}$ salt work as ionic conductors to actuate the dielectric elastomer. The elasticity of the solid electrodes and the ramping velocity of applied triangular voltages are found to influence the residual and maximum strains of a circular actuator. Compared with carbon grease electrodes, solid electrodes constrain electro-actuation deformation and diminish the viscoelastic relaxation of elastomer. Good correlation between experiment and simulation is achieved. These results help the analysis and assessment of performance of dielectric elastomer actuators subject to dynamic electrical loadings.

\section{ACKNOWLEDGEMENTS}

This research is supported by Natural Science Foundation of China (grants 61025002, 11072185, 11372239 and 11021202). ZS acknowledges the support of NSF MRSEC (DMR-0820484) and visiting appointment at the International Center for Applied Mechanics. 


\section{References}

${ }^{1}$ Q. Pei, R. Pelrine, M. Rosenthal, S. Stanford, H. Prahlad, R. Kornbluh, Proc. SPIE 5385, 41 (2004).

Recent progress on electroelastomer artificial muscles and their application for biomimetic robots

${ }^{2}$ H. R. Choi, K. Jung, S. Ryew, J. D. Nam, J. Jeon, J. C. Koo, K. Tanie, IEEE/AAME Trans. Mechatron. 10, 581 (2005).

Biomimetic Soft Actuator: Design, Modeling, Control, and Applications

${ }^{3}$ K. Jung, J. C. Koo, J. D. Nam, Y. K. Lee, and H. R. Choi, Bioinsp. Biomim. 2, S42 (2007).

Artificial annelid robot driven by soft actuators

${ }^{4}$ Jonathan Rossiter, Peter Walters, and Boyko Stoimenov, Proc. SPIE 7287, 72870H (2009).

Printing 3D dielectric elastomer actuators for soft robotics

${ }^{5}$ C. Jordi, S. Michel, and E. Fink, Bioinsp. Biomim. 5, 026007 (2010).

Fish-like propulsion of an airship with planar membrane dielectric elastomer actuators

${ }^{6}$ I. A. Anderson, T. A. Gisby, T. G. McKay, B. M. Obrien, and E. P. Calius, J. Appl. Phys. 112, 041101 (2012).

Multi-functional dielectric elastomer artificial muscles for soft and smart machines

${ }^{7}$ J. Newton, J. Morton, J. Clark, and W. S. Oates, Proc. SPIE 8340, $83400 Z$ (2012).

Modeling and Characterization of Stiffness Controlled Robotic Legs Using Dielectric Elastomers

${ }^{8}$ S. Kim, C. Laschi, and B. Trimmer, Trends Biotechnol. 31, 23 (2013).

Soft robotics: a bioinspired evolution in robotics

${ }^{9}$ P. Brochu, Q. Pei, Macromol. Rapid Commun. 31, 10 (2010).

Advances in Dielectric Elastomers for Actuators and Artificial Muscles

${ }^{10}$ F. Carpi, G. Frediani, S. Turco, and D. De Rossi, Adv. Funct. Mater. 21, 4152-4158 (2011).

${ }^{11}$ S. Son, D. Pugal, T. Hwang, H. R. Choi, J. C. Koo, Y. Lee, K. Kim, and J. D. Nam, Appl. Opt. 51, 2987 (2012).

${ }^{12}$ S. Shian, R. M. Diebold and D. R. Clarke, Optics Express 21, 8669 (2013).

${ }^{13}$ R. Pelrine, R. Kornbluh, J. Joseph, R. Heydt, Q. Pei, and S. Chiba, Mater. Sci. and Eng. C 11, 89 (2000).

High-field deformation of elastomeric dielectrics for actuators

${ }^{14}$ R. Pelrine, R. Kornbluh, Q. Pei and J. Joseph, Science 287, 836 (2000).

High-speed electrically actuated elastomers with strain greater than $100 \%$

${ }^{15}$ Xuequn Zhang, Christiane Löwe, and Michael Wissler, Adv. Eng. Mater. 7, 361 (2005).

Dielectric elastomers in actuator technology

${ }^{16}$ Ravi Shankar, Tushar K. Ghosh, and Richard J. Spontak, Soft Matter 3, 1116 (2007).

Dielectric elastomers as next-generation polymeric actuators

${ }^{17}$ M. Matysek, P. Lotz, and H. F. Schlaak, Proc. SPIE 7287, 72871D (2009).

Tactile display with dielectric multilayer elastomer actuators

${ }^{18}$ F. Carpi, S. Bauer and D. De Rossi, Science 330, 1759 (2010).

${ }^{19}$ R. Sarban, R. Jones, B. Mace, and E. Rustighi, Mech. Sys. Signal Process. 25, 2879 (2011).

A tubular dielectric elastomer actuator: Fabrication, characterization and active vibration isolation

${ }^{20}$ A. D. Poole, J. D. Booker, C. L. Wishart, N. McNeill, and P. H. Mellor, IEEE/AAME Trans. Mechatron. 17, 525 (2012). 
Performance of a Prototype Traveling-Wave Actuator Made From a Dielectric Elastomer

${ }^{21}$ S. Kim, B. C. Kim, J. C. Koo, H. R. Choi, and H. Moon, Proc. SPIE 8340, 83402H (2012). Dual-axis hybrid tactile sensor

${ }^{22}$ S. Laflamme, M. Kollosche, J. J. Connor, and G. Kofod, Struct. Control Health Monit. 19, 70 (2012). Soft capacitive sensor for structural health monitoring of large-scale systems

${ }^{23}$ T. McKay, B. O’Brien, E. Calius, and I. Anderson, Appl. Phys. Lett. 97, 062911 ( 2010). An integrated, self-priming dielectric elastomer generator

${ }^{24}$ J. Huang, S. Shian, Z. Suo, and D. R. Clarke, Adv. Funct. Mater., 2013. Maximizing the Energy Density of Dielectric Elastomer Generators Using Equi-Biaxial Loading

${ }^{25}$ S. Chiba, M. Waki, R. Kornbluh, and R. Pelrine, Proc. SPIE 6927, 692715, (2008). Innovative Power Generators for Energy Harvesting Using Electroactive Polymer Artificial Muscles

${ }^{26}$ T. McKay, B. O’Brien, E. Calius, and I. Anderson, Appl. Phys. Lett. 98, 142903 (2011). Soft generators using dielectric elastomers

${ }^{27}$ P. Larsen, G. Kofod, M.H. Shridhar, M. Benslimane, and P. Gravesen, Proc. SPIE 4695, 158 (2002). Performance of dielectric elastomer actuators and materials

${ }^{28}$ S. Bauer, M.Paajanen, Proc. SPIE 6168, 61682K (2006). Electromechanical characterization and measurement protocol for dielectric elastomer actuators

${ }^{29}$ J. W. Fox, N.C. Goulbourne, J. Mech. Phys. Solids 56, 2669 (2008). On the dynamic electromechanical loading of dielectric elastomer membranes

${ }^{30}$ A York, J Dunn, and S Seelecke, Smart Mater. Struct. 19, 094014 (2010). Experimental characterization of the hysteretic and rate-dependent electromechanical behavior of dielectric electro-active polymer actuators

${ }^{31}$ C. C. Foo, S. Q. Cai, S. J. A. Koh, S. Bauer, and Z. G. Suo, J. Appl. Phys. 111, 034102 (2012).

Model of dissipative dielectric elastomers

${ }^{32}$ C. C. Foo, S. J. A. Koh, C. Keplinger, R. Kaltseis, S. Bauer, and Z. G. Suo, J. Appl. Phys. 111,094107 (2012).

Performance of dissipative dielectric elastomer generators

${ }^{33}$ M. Bozlar, C. Punckt, S. Korkut, J. Zhu, C. C. Foo, Z. G. Suo, and I. A. Aksay, Appl. Phys. Lett. 101, 091907 (2012).

Dielectric elastomer actuators with elastomeric electrodes

${ }^{34}$ H. S. Park, T. D. Nguyen, Soft Matter 9, 1031 (2013).

Viscoelastic effects on electromechanical instabilities in dielectric elastomers

${ }^{35}$ H. M. Wang, M. Lei, and S. Q. Cai, J. Appl. Phys. 113, 213508 (2013).

Viscoelastic deformation of a dielectric elastomer membrane subject to electromechanical loads

${ }^{36}$ F. Carpi, P. Chiarelli, A. Mazzoldi, and Danilo De Rossi, Sens. Actuators, A 107, 85 (2003).

Electromechanical characterisation of dielectric elastomer planar actuators: comparative evaluation of different electrode materials and different counterloads

${ }^{37}$ S. Rosset, M. Niklaus, P. Dubois, and H. R. Shea, Adv. Funct. Mater. 19, 47 (2009). Metal Ion Implantation for the Fabrication of Stretchable Electrodes on Elastomers

${ }^{38}$ D. C. Hyun, M. Park, C. J. Park, B. Kim, Y. Xia, J. H. Hur, J. M. Kim, J. J. Park, and U. Jeong, Adv. Mater. 23, 2946 (2011). 
${ }^{39}$ S. Michel, B. T. Chu, S. Grimm, F. A. Nüesch, A. Borgschulte and D. M. Opris, J. Mater. Chem. 22, 20736 (2012).

Self-healing electrodes for dielectric elastomer actuators

${ }^{40}$ S. Rosset, H. R. Shea, Appl. Phys. A 110, 281 (2013).

Flexible and stretchable electrodes for dielectric elastomer actuators

${ }^{41}$ C. Keplinger, J. Y. Sun, C. C. Foo, P. Rothemund, G. M. Whitesides, and Z. G. Suo, Science 341, 984 (2013).

${ }^{42}$ Baohong's paper.

${ }^{43}$ W. Hong, J. Mech. Phys. Solids 59, 637 (2011).

${ }^{44}$ A. N.Gent, Rubber Chemy. Tech. 69, 59 (1996).

A New Constitutive Relation for Rubber 


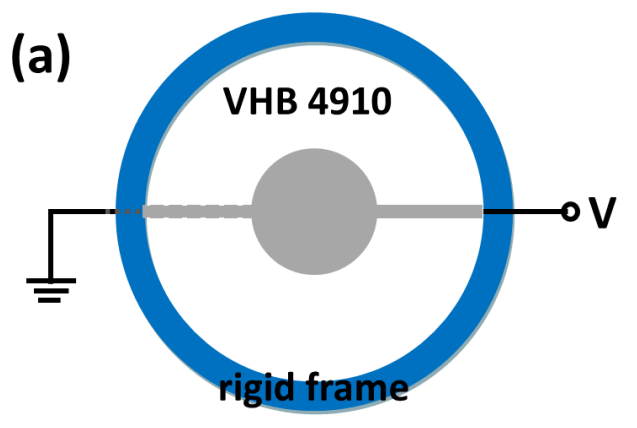

(b)
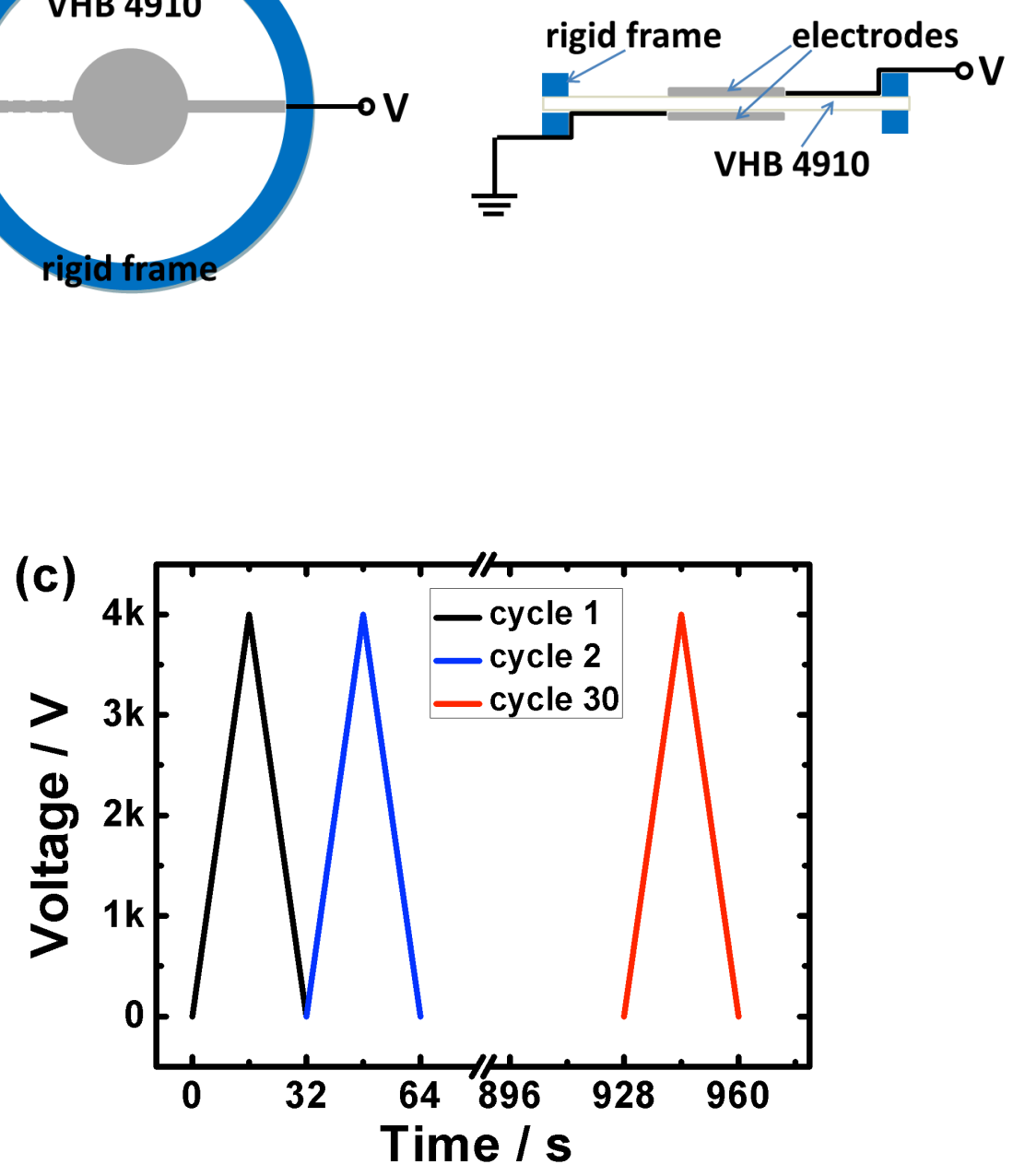

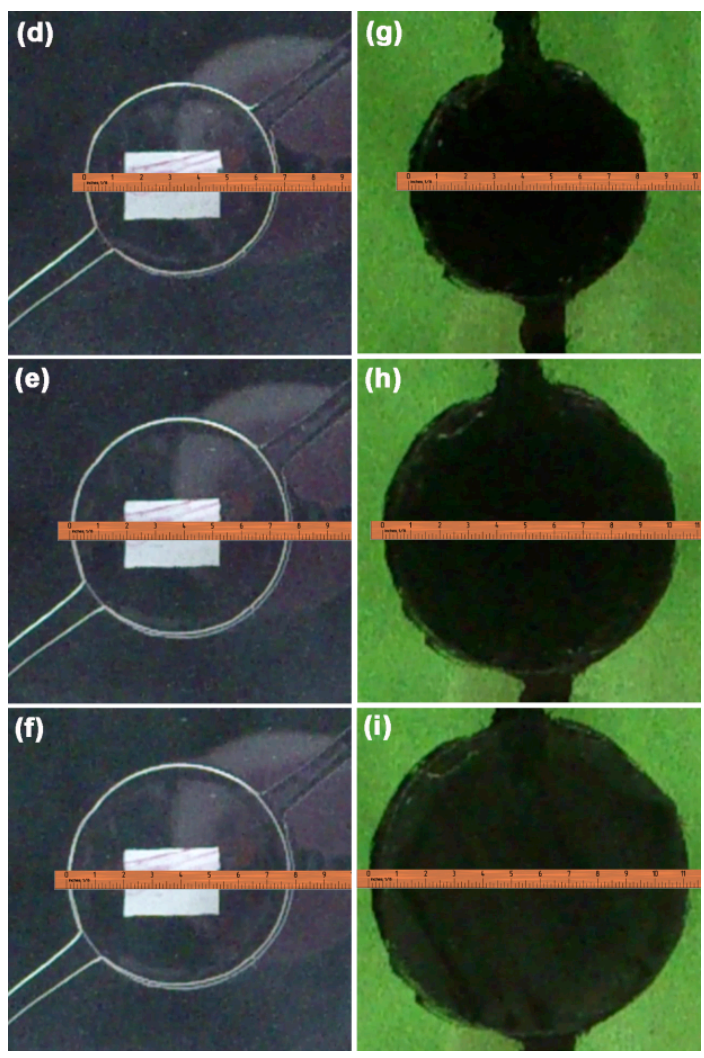

Figure 1. Experimental setup of a circular DE actuator. (a) and (b): schematics of the top and side views of the setup; (c): applied cyclic triangular waveform voltage in experiment; (d), (e) and (f): photos of DE with hydrogel electrodes corresponding to voltage-off state, voltage-on states for cycle 1 and cycle 30, respectively; (g), (h) and (i): photos of DE with carbon grease electrodes corresponding to voltage-off state, voltage-on states for cycle 1 and cycle 30, respectively. The hydrogel electrodes are stretchable and transparent. The white scale and the ruler are used for calculating the areal strain. 

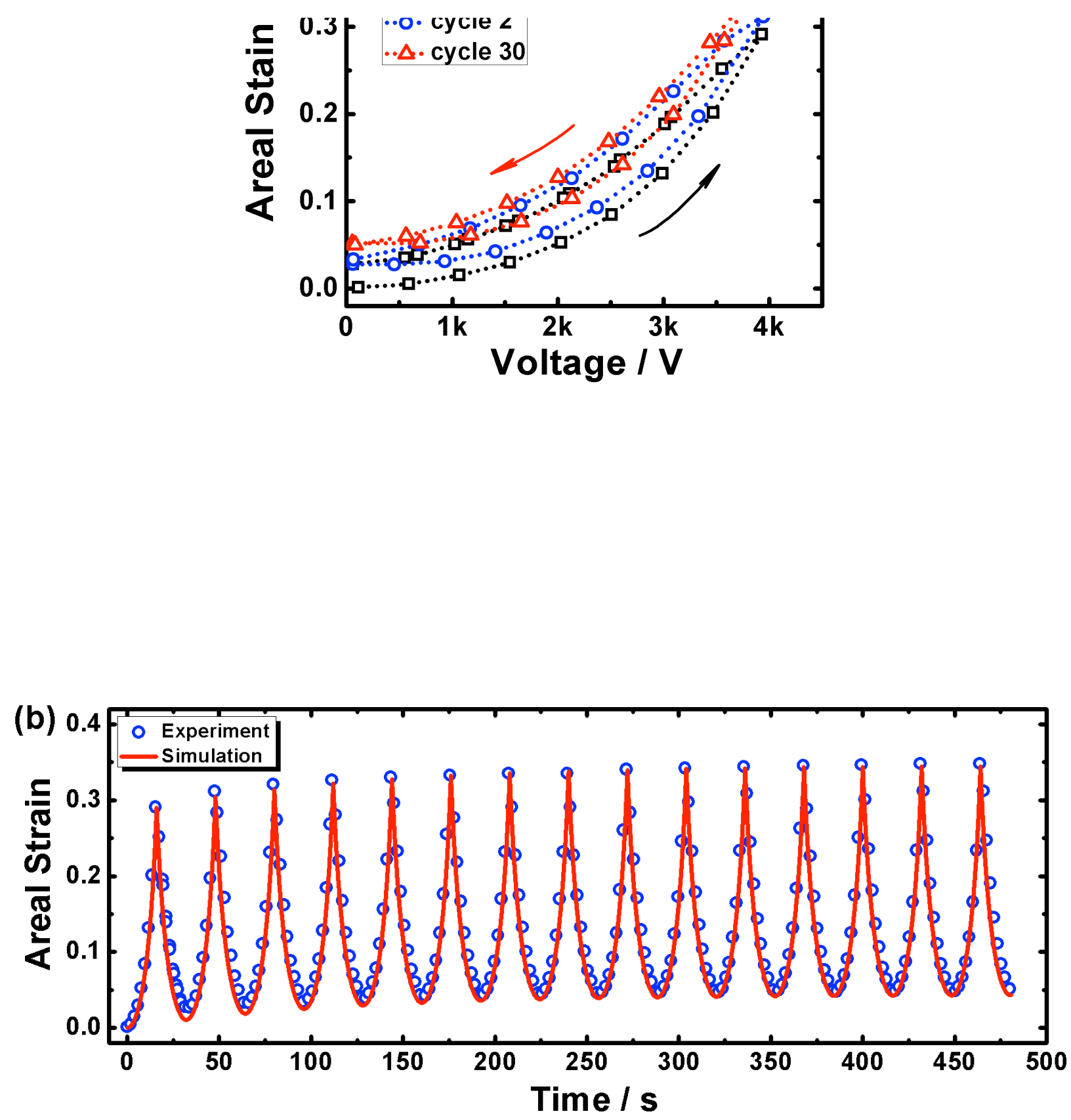

Figure 2. Viscoelastic performance of a circular DE with hydrogel electrodes under supply of a series of cyclic voltages. (a): Areal strain versus voltage for three typical cycles (cycles 1, 2 and 30, respectively; ramping velocity: $250 \mathrm{O} / \mathrm{s}$ ); (b): Comparison of experiment and simulation of the time history of areal strain (open blue circle: experiment, red solid line: simulation; ramping velocity: $250 \mathrm{~V} / \mathrm{s})$. 

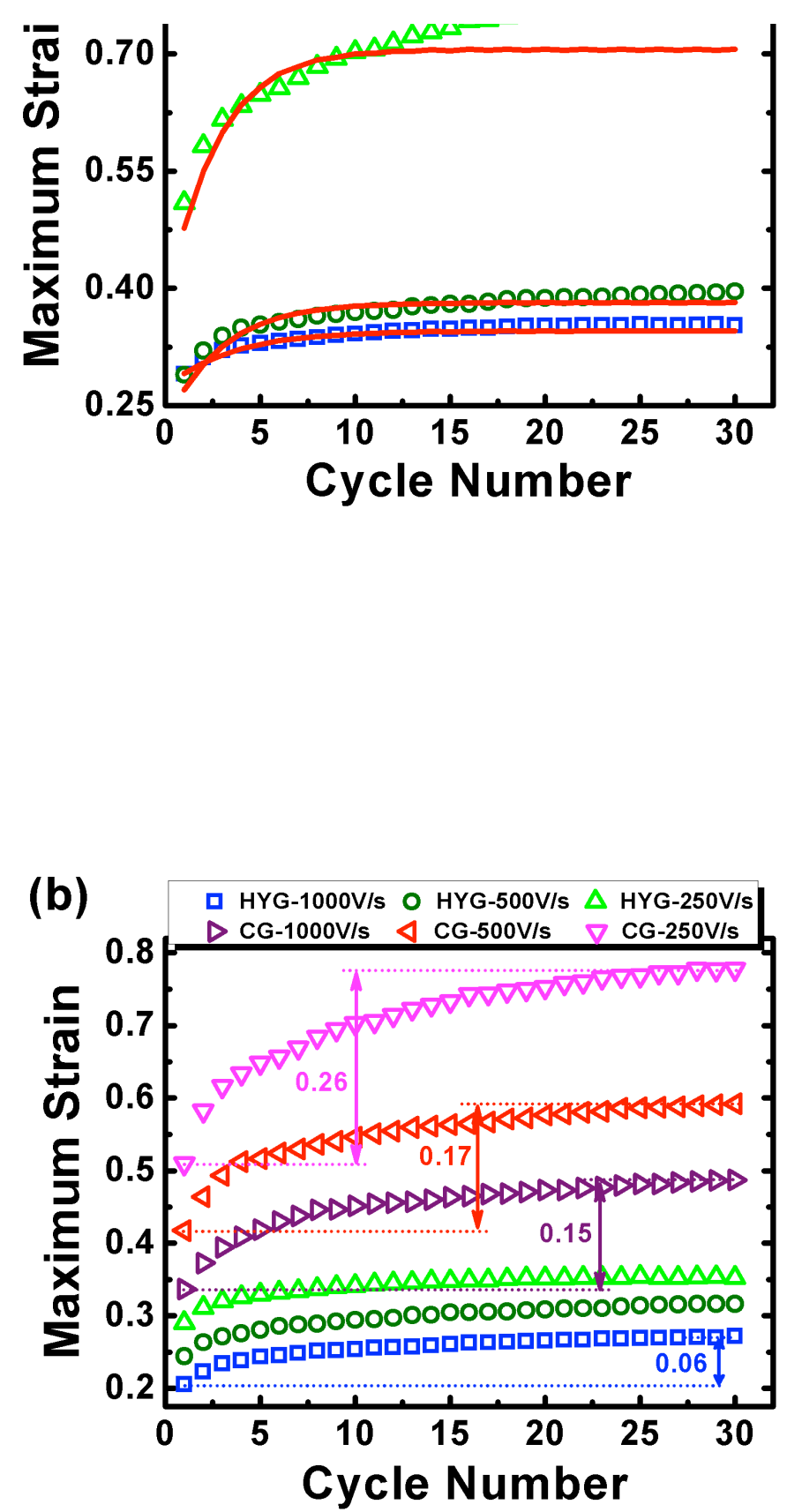

Figure 3. Effects of elastomeric electrodes on the maximum areal strain of DE actuators. (a): Comparison of cyclic maximum strains for carbon grease electrodes and $0.5 \mathrm{~mm}$ thick hydrogel electrodes. The applied ramping velocity is fixed to $250 \mathrm{~V} / \mathrm{s}$. The markers are experimental results while the red solid line represents the simulation result; (b)The effects of ramping velocity on the evolution of cyclic maximum strains. The amplitude of applied voltage is fixed to be $4 \mathrm{kV}$ and the ramping velocities are varied to be $250 \mathrm{~V} / \mathrm{s}, 500 \mathrm{~V} / \mathrm{s}$ and $1000 \mathrm{~V} / \mathrm{s}$, respectively. The marked shift is the difference between the first and the steady-state maximum strain. HYG 
denotes hydrogel electrodes while CG denotes carbon grease electrodes. 